\title{
Analgesic Effect of Ultrasound-Guided Preoperative Unilateral Lateral Quadratus Lumborum Block for Laparoscopic Nephrectomy: A Randomized, Double-Blinded, Controlled Trial
}

This article was published in the following Dove Press journal:

Journal of Pain Research

\section{Kyung-Hwa Kwak' \\ Seung Ik Baek' \\ Jay Kyoung Kim' \\ Tae-Hwan Kim ${ }^{2}$ \\ Jinseok Yeo (D)}

'Department of Anesthesiology and Pain Medicine, Kyungpook National University Chilgok Hospital, Kyungpook National University School of Medicine, Daegu, Korea; ${ }^{2}$ Department of Urology, Kyungpook National University Chilgok Hospital, Kyungpook National University School of Medicine, Daegu, Korea
Correspondence: Jinseok Yeo Department of Anesthesiology and Pain Medicine, Kyungpook National Chilgok Hospital, Kyungpook National University School of Medicine, Daegu, Korea

Tel +82 53-200-2644

Fax +82 53-200-2027

Email painfree@gmail.com
Purpose: The quadratus lumborum block (QLB) is a relatively new regional analgesic technique that could provide analgesia to the abdominal wall and reduce postoperative opioid consumption. We investigated the opioid-sparing effect of a unilateral lateral QLB in laparoscopic nephrectomy. Patients and Methods: A total of 60 patients undergoing laparoscopic nephrectomy were included in the study. Patients were randomized into two groups as QLB and control group. QLB group received QLB with $25 \mathrm{~mL} 0.25 \%$ ropivacaine, and the control group received $25 \mathrm{~mL} 0.9 \%$ saline at anterolateral border of quadratus lumborum muscle preoperatively. Opioid consumption and the pain intensity at rest and on movement were measured at $2 \mathrm{nd}$, 6th, 24th, and 48th hour postoperatively. We also assessed the time to first flatus to measure the extent of paralytic ileus and the quality of recovery-15 (QoR-15) questionnaire.

Results: Postoperative opioid consumption was significantly lower in the QLB group than in the control group at 6,24 , and $48 \mathrm{~h}$ after surgery $(P<0.05)$. The pain intensity at rest and on movement was significantly lower in the QLB group than in the control group during the first 24 hours after surgery $(P<0.05)$. The incidence of postoperative nausea and vomiting, time to first flatus, and QoR-15 score did not show significant differences.

Conclusion: Preoperative unilateral QLB successfully decreased postoperative pain and opioid consumption after laparoscopic nephrectomy and could be an option for analgesia after laparoscopic nephrectomy.

Keywords: quadratus lumborum block, laparoscopic nephrectomy, postoperative pain, opioid consumption, ultrasound-guided block

\section{Plain Language Summary}

- Inadequate pain control after nephrectomy is still a problem. It decreases patients' satisfaction with recovery and increases postoperative complications.

- Quadratus lumborum block (QLB) is a relatively new technique to provide analgesia to the abdominal wall, but there is limited evidence of analgesic effects of QLB in laparoscopic nephrectomy.

- Unilateral preoperative QLB reduces postoperative opioid requirements and pain in laparoscopic nephrectomy patients.

\section{Introduction}

Postoperative pain control is still a significant challenge in medical practice, and insufficient postoperative pain control remains high. ${ }^{1}$ Inadequate pain control could 
decrease a patient's satisfaction, delay postoperative ambulation, increase the incidence of pulmonary and cardiac complications, and cause the development of chronic postoperative pain. Laparoscopic surgical techniques could decrease postoperative pain and opioid consumption. ${ }^{2}$

Postoperative pain control with an opioid is the gold standard; however, it increases the incidence of opioidrelated adverse events such as dizziness, nausea, vomiting, constipation, and respiratory depression. ${ }^{3}$ Therefore, minimizing opioid use and opioid side effects is essential for postoperative pain control.

Regional analgesia is a useful method for minimizing opioid use and postoperative pain. It also could reduce morbidity and mortality after surgery. Epidural analgesia has excellent analgesic profiles and decreases postoperative complications. Still, it could produce procedure-related complications such as dural puncture, hypotension, postoperative urinary retention, and delayed mobilization. ${ }^{4}$ Recently, interfascial plane block is applied for postoperative pain control in abdominal surgery. Quadratus lumborum block (QLB) is a relatively new interfascial plane block technique that injects local anesthetics adjacent to the quadratus lumborum muscle. QLB has 4 different approaches anterior, intramuscular, lateral, and posterior. ${ }^{5}$ The anterior QLB injects local anesthetics between the quadratus lumborum (QL) muscle and psoas muscle. Intramuscular QLB injects local anesthetics within the QL muscle. The lateral QLB injects the local anesthetics at the anterolateral border of the QL muscle (deep to transversalis fascia). The posterior QLB injects the local anesthetics at the posterior to the QL muscle. It provides analgesia to the abdominal wall. It potentially has a visceral analgesic effect and offers an extended sensory block area than the transversus abdominis plane block. ${ }^{6,7}$ But there is limited evidence of analgesic effects of QLB in laparoscopic nephrectomy.

We hypothesized that a single unilateral preoperative lateral QLB could reduce pain and opioid consumption after laparoscopic nephrectomy. To evaluate the potential analgesic benefit of the lateral QLB, we conducted a randomized, double-blinded, placebo-controlled study to assess opioid use (primary endpoint) and pain scores (secondary endpoint) in the 48 hours following laparoscopic nephrectomy.

\section{Patients and Methods}

\section{Patients}

The study was approved by the Kyungpook National University Chilgok Hospital review board and registered at
CRIS (cris.nih.go.kr: KCT0001973). We conducted this study in accordance with "Helsinki Declaration (version 19 October 2013)". Patients who were scheduled for laparoscopic nephrectomy between July 2016 and July 2017 were considered for entry into the trial. A member of the research team contacted the patients, explained the study protocol, and obtained informed consent for participation in the study. We included patients aged 20 to 75 years old who were categorized as American Society of Anesthesiologists (ASA) Physical Status (PS) I and II. We excluded patients who had an analgesic within 24 hours, asthma or chronic obstructive pulmonary disorder, chronic pain syndrome, a history of allergy to local anesthetics or systemic opioids, substance use disorder, or sleep apnea.

\section{Protocol}

We randomly allocated patients to the QLB group $(\mathrm{n}=30)$ or control group $(n=30)$ in a 1:1 allocation ratio by block randomization with blocks of size 4 . We sealed the allocation results in opaque envelopes that were not opened until the day of surgery. The patients, anesthesiologists, and surgeons providing postoperative care were blinded to the group assignment.

During the preanesthetic visit, the patients were trained to record their postoperative pain on the Numeric Rating Pain Scale (NRPS; "0" represents "no pain" and "10" represents "the worst pain imaginable"). On the day of surgery, all patients received general anesthesia using standard ASA monitors, bispectral index (A-2000 BIS ${ }^{\mathrm{TM}}$ monitor, Aspect Medical Systems, Inc., Natick, MA, USA) and continuous blood pressure monitoring using radial artery catheter in the dependent arm. Propofol $(1-2 \mathrm{mg} / \mathrm{kg}$ ) and rocuronium $(0.4 \mathrm{mg} / \mathrm{kg})$ were administered for the induction of anesthesia. We used Desflurane (4-8\%) and remifentanil (2-4 ng/ $\mathrm{mL}$ using a target-controlled infusion pump) to keep BIS score between 40 and 60 and blood pressure within $20 \%$ of preoperative value during anesthesia. Additional doses of rocuronium were administered as needed to maintain muscle relaxation. After the induction of anesthesia, the patients were placed in the semi-lateral decubitus position, and an ultrasound transducer was placed in the posterior axillary line between the subcostal margin and the iliac crest. As the probe moved posteriorly, the lumbar interfascial triangle and quadratus lumborum muscle appeared. A 22-gauge Tuohy needle was placed at the anterolateral border of the QL muscle using an in-plane method (Figure 1). The correct position of the needle tip was confirmed by injecting $3 \mathrm{~mL}$ of saline to separate the fascial layers. A nurse who played 


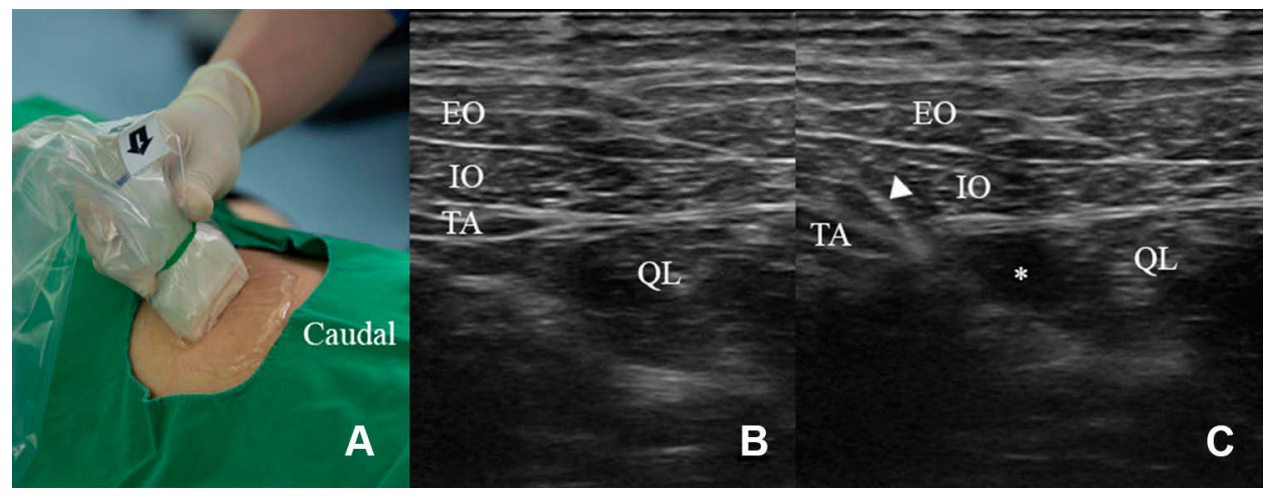

Figure I (A) Patient was positioned in lateral decubitus position. A high-frequency linear probe attached in the area of posterior to triangle of Petit. (B) Preinjection and (C) postinjection images of quadratus lumborum blocks. Triangle indicates needle. Asterisk represents the spread of local anesthetics.

Abbreviations: EO, external oblique muscle; IO, internal oblique muscle; TA, transversus abdominis muscle; QL, quadratus lumborum muscle.

no other role in the study prepared an injection according to the group allocation. After the negative aspiration of blood, patients in the control group received $25 \mathrm{~mL}$ of normal saline, and patients in the QLB group received $25 \mathrm{~mL}$ of $0.25 \%$ ropivacaine. No additional analgesics were injected during surgery.

At the end of the surgery, a patient-controlled analgesia device (AIM Plus; Hospira, Lake Forest, IL, USA) was connected which was set to deliver a bolus of $16 \mu \mathrm{g}$ of fentanyl with a lockout time of 5 minutes without baseline infusion. Anesthesia was discontinued, and the patient was extubated and transferred to the postanesthetic care unit. If the patient complained of severe pain (NRPS $>5$ for 30 minutes), tramadol $(50 \mathrm{mg})$ was given intravenously as rescue analgesia. A research assistant who was blinded to the group allocation monitored the fentanyl consumption and the pain intensity at rest and on movement at 2, 6, 24, and 48 hours after the operation. We also assessed the time to first flatus by asking the patients to measure the extent of paralytic ileus, and the quality of recovery-15 (QoR-15) questionnaire was given 48 hours after the operation. Any adverse events were recorded, such as local anesthetics toxicity, allergy, visceral organ injury, postoperative nausea and vomiting, postoperative bleeding, and delirium.

\section{Sample Size Calculation and Statistical Analyses}

The primary outcome in this study was opioid consumption during the first 24 hours after surgery. The secondary outcomes were pain intensities, quality of recovery, and side effects associated with opioid consumption. We assumed that a clinically significant reduction in opioid use would be $30 \%{ }^{8}$ Based on our pilot study, the mean 24 - hour fentanyl consumption was $1200 \mu \mathrm{g}$, with a standard deviation of $350 \mu \mathrm{g}$ in the control group. We included 26 patients per group to provide $80 \%$ power and a significance level $(\alpha)$ of 0.05 . To minimize the effect of data loss, we selected 30 patients for each group in this study.

Statistical analysis was performed using SPSS software version 21.0 (IBM Corporation, Armonk, NY, USA). Categorical data (expressed as raw data and frequencies) were analyzed by the $\chi^{2}$ test or Fisher's exact test. Continuous data (presented as the mean \pm standard deviation) were analyzed by Student's $t$-test if normally distributed. The results were considered significant when $P<0.05$ (2-sided).

\section{Results}

A total of 85 patients were assessed; 60 patients met the inclusion criteria and agreed to participate in the study. The Consolidated Standards of Reporting Trials (CONSORT) diagram is shown in Figure 2. All 60 patients completed the study. Demographic and perioperative data are shown in Table 1. No significant differences were observed between the two groups in terms of age, sex, body mass index, ASA PS, duration of anesthesia, and surgery. Cumulative postoperative opioid consumption (primary outcome) was significantly lower in the QLB group than in the placebo group at $6 \mathrm{~h}, 24 \mathrm{~h}$, and $48 \mathrm{~h}$ after surgery $(P<0.05$, Table 2$)$. Eight patients received rescue analgesics in placebo groups, and three patients received rescue analgesics in the QLB group. But the number of patients requiring rescue analgesia was not statistically significant between the two groups. Postoperative pain at rest and on movement was significantly lower in the QLB group than in the placebo 


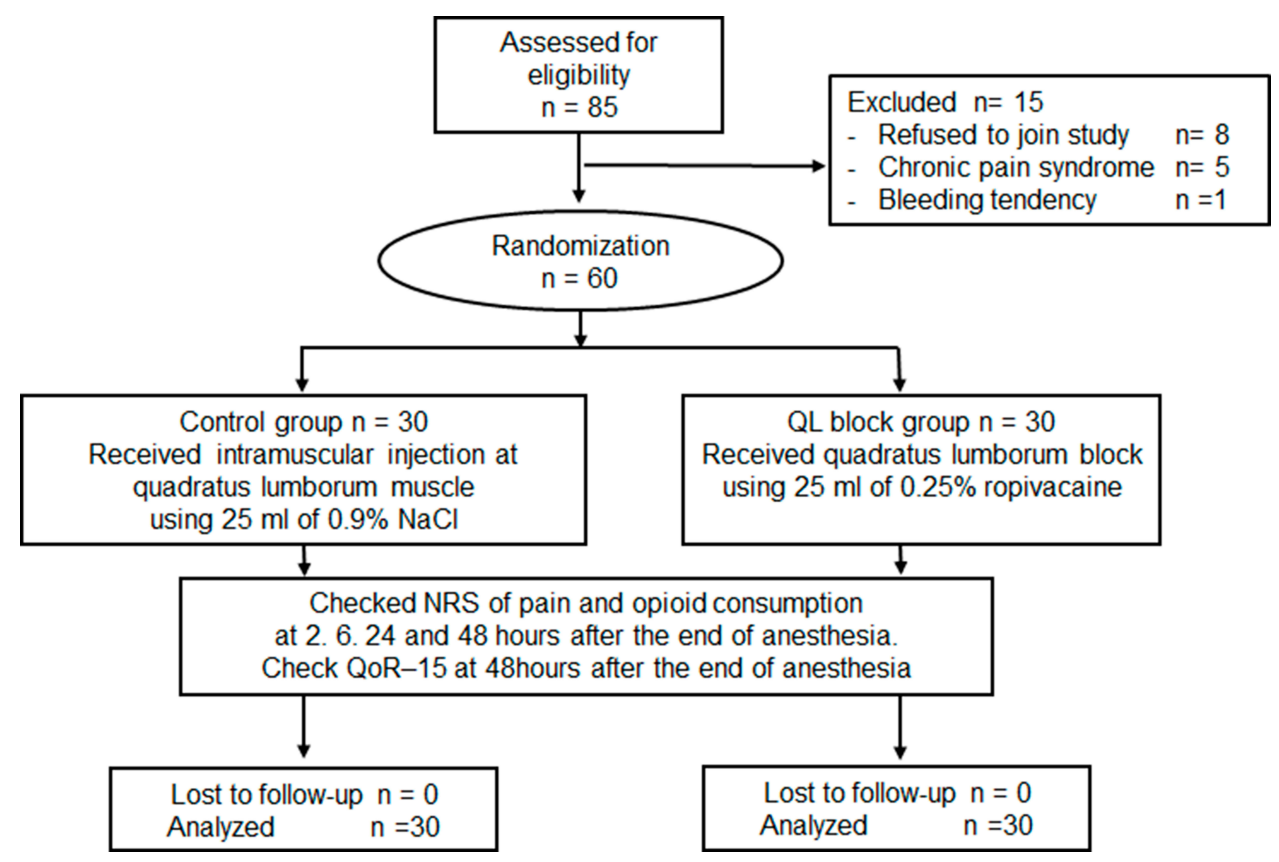

Figure 2 The Consolidated Standards of Reporting Trials (CONSORT) flowchart of the study.

Abbreviations: QL, quadratus lumborum; NRS, numeric rating scale; QoR-15, questionnaire of quality of recovery with 15 items.

group up to the 24th postoperative hour $(P<0.05$, Figure 3). The incidence of postoperative nausea and vomiting, time to first flatus, and QoR-15 score did not show significant differences (Table 3). There were no serious complications in any patients, including local anesthetic toxicity, allergy, visceral organ injury, postoperative bleeding, and postoperative infection.

\section{Discussion}

This study demonstrated that a preoperative unilateral QLB reduced postoperative opioid consumption and decreased pain intensity after laparoscopic nephrectomy. But it did not improve the quality of recovery. Adequate and safe postoperative analgesia is essential in postoperative care. However, postoperative opioid use to control

Table I Patient Characteristics and Perioperative Data

\begin{tabular}{|c|c|c|c|}
\hline \multirow[t]{2}{*}{ Group } & \multirow{2}{*}{$\begin{array}{l}\text { Control } \\
(n=30)\end{array}$} & \multirow{2}{*}{$\begin{array}{l}\text { QLB } \\
(n=30)\end{array}$} & \multirow[t]{2}{*}{$P$ value } \\
\hline & & & \\
\hline Age (yr.) & $56.1 \pm 10.7$ & $55.8 \pm 11.6$ & 0.92 \\
\hline Sex & & & 0.28 \\
\hline Male & $21(70 \%)$ & $17(56.6 \%)$ & \\
\hline Female & $9(30 \%)$ & $13(43.3 \%)$ & \\
\hline BMI, $\mathrm{kg} / \mathrm{m}^{2}$ & $24.86 \pm 4.07$ & $24.98 \pm 2.59$ & 0.89 \\
\hline ASA Physical status class & & & 0.32 \\
\hline I* & $7(23.3 \%)$ & $4(13.4 \%)$ & \\
\hline $\mathrm{II}^{\dagger}$ & $23(76.7 \%)$ & $26(86.7 \%)$ & \\
\hline Duration of operation (min) & $171.3 \pm 53.9$ & $148.8 \pm 29.8$ & 0.05 \\
\hline Duration of anesthesia, min, mean $\pm S D$ & $211.8 \pm 63.3$ & $188.4 \pm 29.7$ & 0.08 \\
\hline Intraoperative fluid, $\mathrm{mL}$, mean $\pm \mathrm{SD}$ & $1324.0 \pm 344.9$ & $1363.3 \pm 356.9$ & 0.58 \\
\hline Decreased hemoglobin concentration during operation, $g / d L$, mean $\pm S D$ & $1.08 \pm 0.82$ & $1.26 \pm 1.19$ & 0.51 \\
\hline
\end{tabular}

Note: Data are presented as mean \pm standard deviation or number (\%). *Class I, normal healthy patient. ${ }^{\dagger}$ Class II, patient with mild systemic disease. Abbreviations: ASA, American Society of Anesthesiologists; BMI, Body Mass Index; QLB, quadratus lumborum block. 
Table 2 Fentanyl Consumption and Rescue Analgesics Use in the Placebo Group and QLB Group

\begin{tabular}{|l|l|l|l|}
\hline Variables & Control & QLB & \multirow{2}{*}{ P value } \\
\cline { 2 - 3 } & $\mathbf{( n = 3 0 )}$ & $\mathbf{( n = 3 0 )}$ & \\
\hline $\begin{array}{l}\text { Fentanyl } \\
\text { consumption, } \mu \mathrm{g} \\
2 \mathrm{~h}\end{array}$ & & & \\
$6 \mathrm{~h}$ & $259.5 \pm 139.9$ & $220.5 \pm 85.8$ & 0.197 \\
$24 \mathrm{~h}$ & $512.3 \pm 301.1$ & $341.9 \pm 158.1$ & 0.010 \\
$48 \mathrm{~h}$ & $982.2 \pm 481.1$ & $599.5 \pm 271.0$ & 0.001 \\
\hline $\begin{array}{l}\text { Number of patients } \\
\text { requiring rescue }\end{array}$ & 8 & $808.9 \pm 416.3$ & 0.000 \\
analgesia, $\mathrm{n}$ & & 3 & 0.405 \\
\hline
\end{tabular}

Note: Data are presented as means \pm standard deviation or number of patients. Abbreviation: QLB, quadratus lumborum block.

pain leads to side effects such as nausea, vomiting, dizziness, and paralytic ileus, which prevent rapid recovery. The regional analgesic technique as a component of multimodal analgesia could reduce postoperative pain and opioid consumption. ${ }^{9}$ The ultrasound-guided interfascial plane block has been introduced for postoperative pain control. It has a short procedure time and less severe complications. Transversus abdominis plane (TAP) block is a commonly used interfascial plane block for analgesia of the abdominal wall. It provides analgesia to the T10 T12 dermatomes only; thus, it does not affect the upper abdomen. $^{10}$

QLB is a relatively new interfascial plane block that provides analgesia to the abdominal wall and visceral analgesia. QLB has four different approach methods: lateral, posterior, anterior, and intramuscular. The lateral approach applies local anesthetics on the lateral side of the quadratus lumborum muscle, which contacts with the lateral margin of the transverse fascia. ${ }^{11}$ It could have a lesser chance of having a penetration injury than the anterior approach because the needle tip was placed between quadratus lumborum muscle and transverse fascia. The lateral QLB provides analgesia to the T7-L1 dermatomes. ${ }^{5}$ Laparoscopic nephrectomy requires a port to remove the excised specimen and three incisions for working ports (Figure 4). The port for the excised specimen was made by a $5-7 \mathrm{~cm}$ skin incision, located halfway between the xiphoid and the umbilicus in laparoscopic nephrectomy, and working ports were situated below the ipsilateral costal margin. The lateral QLB could provide analgesia to all incisions in laparoscopic nephrectomy.
Pain at rest

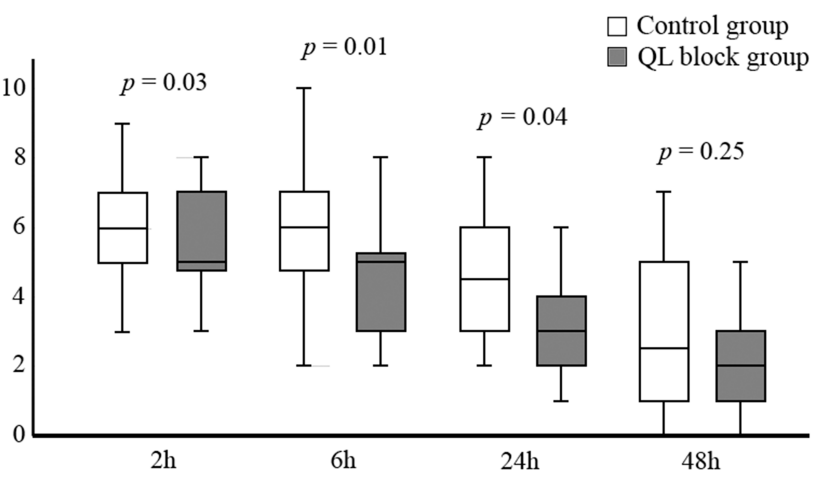

Pain during movement

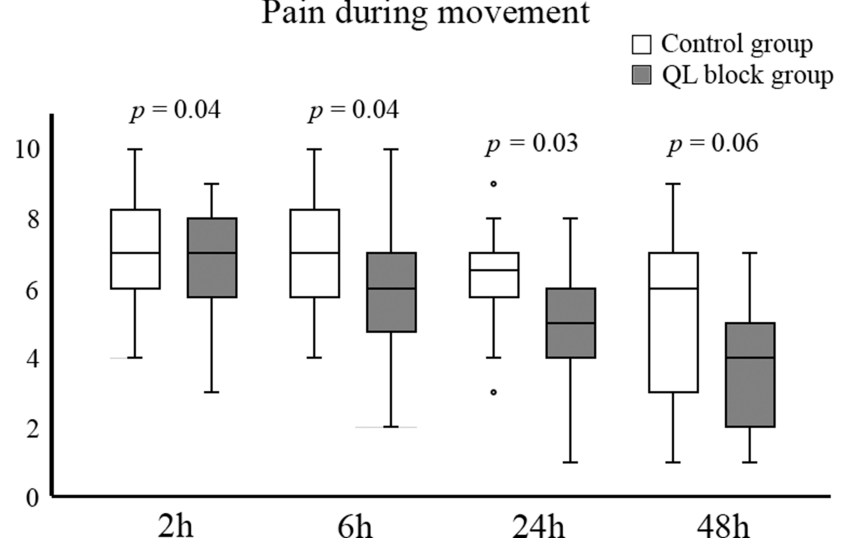

Figure 3 Pain intensities in the control group and QL block group at 2, 6, 24, and $48 \mathrm{~h}$ after surgery. The box plot demonstrates the median with interquartile range (IQR). The whiskers represent I.5 times the IQR or the limits of the numeric rating scale of pain $(0-10)$. Outliers are data beyond theses values and denoted by small circles.

Abbreviation: QL, quadratus lumborum.

QLB usually applied bilaterally in abdominal surgeries, such as cesarian section, laparoscopic gynecological surgery, and laparoscopic cholecystectomy. ${ }^{7,11,12}$ It successfully decreases postoperative pain and analgesics consumption after abdominal surgery. The unilateral interfascial block is

Table 3 Incidences of PONV and Recovery Parameters

\begin{tabular}{|l|l|l|l|}
\hline Variables & Control & QL block & \multirow{2}{*}{ P value } \\
\cline { 2 - 3 } & $(\mathbf{n = 3 0 )}$ & $\mathbf{( n = 3 0 )}$ & \\
\hline Incidence of PONV & & & \\
Overall & $10(33.3 \%)$ & $6(20 \%)$ & 0.243 \\
Less than 6h & $9(30 \%)$ & $6(20 \%)$ & 0.371 \\
6-48h & $6(20 \%)$ & $1(3.3 \%)$ & 0.044 \\
Time to first flatus, $\mathrm{h}$ & $36.2 \pm 8.0$ & $38.0 \pm 7.0$ & $0.35 \mathrm{I}$ \\
QoR-15 & $86.3 \pm 20.8$ & $90.0 \pm 19.3$ & 0.471 \\
\hline
\end{tabular}

Note: Data were presented as means \pm standard deviation or number (\%). Abbreviations: PONV, postoperative nausea and vomiting, QLB, quadratus lumborum block. 


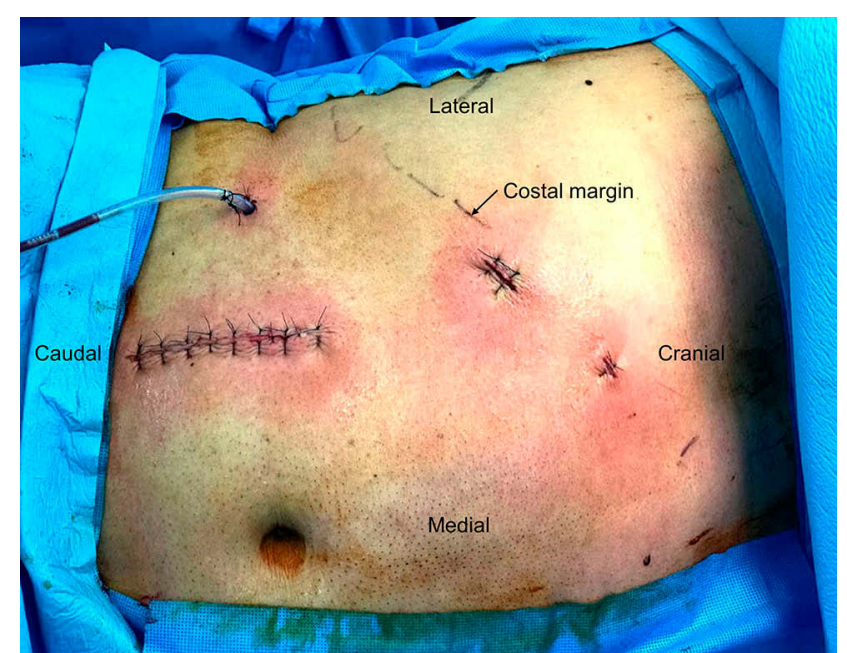

Figure 4 The incisions in the laparoscopic nephrectomy. Our laparoscopic nephrectomy has one paramedian incision for removing excised specimen and three working ports, which lie on the ipsilateral subcostal line.

not common in abdominal surgery. But nephrectomy and laparoscopic nephrectomy usually have unilateral abdominal incisions, so unilateral interfascial block can be applied. Three studies investigated the analgesic effect of the unilateral interfascial plane block in nephrectomy. ${ }^{13,15}$ One study showed postoperative unilateral TAP block reduces pain when compared placebo, ${ }^{13}$ but the other study showed preoperative unilateral TAP block does not decrease pain when compared to local infiltration. ${ }^{14}$ TAP block does not provide the analgesic effect to the upper abdomen, so it has a limited analgesic effect in nephrectomy. ${ }^{10}$ We thought that the limited analgesic effect of the TAP block in the nephrectomy did not show a superior analgesic effect than local anesthetics infiltration. Transmuscular QLB reduced postoperative opioid consumption, postoperative pain, the incidence of rescue analgesics uses, recovery of intestinal function and PONV in laparoscopic nephrectomy. ${ }^{15}$ Lateral QLB in our study also reduced postoperative pain and postoperative opioid consumption. But our study did not decrease the incidence of rescue analgesics use, time to recovery of intestinal function, and PONV. These results are similar to those form other studies comparing transmuscular QLB and posterior QLB in hernia surgery. ${ }^{16,17}$ These results suggest transmuscular QLB could provide more extensive analgesia than lateral QLB. To confirm the differences between the two methods, the direct comparison of the transmuscular QLB and lateral QLB will be required in nephrectomy.

The actual mechanism of analgesia of the QLB is still unknown. Initially, local anesthetic spread along the thoracolumbar fascia (TLF) into the paravertebral space, the celiac ganglion, and the sympathetic chain were suggested as a mechanism for both somatic and visceral analgesia in a QLB. However, the volume of local anesthetics analgesics that reached the paravertebral space was found to be too small in a magnetic resonance imaging study and cadaver study. ${ }^{6,18}$ Recently, the spread of local anesthetics to the TLF is suggested to be the basis for analgesia. ${ }^{5}$ The TLF envelopes the back muscles from the sacral to the thoracic region and consists of the anterior, middle, and posterior layers. ${ }^{19}$ The TLF has sensory nerves that flow in the superficial layer with adjacent connective tissues. ${ }^{20}$

Furthermore, the superficial layer of the TLF has mechanoreceptors, pain receptors, and a network of sympathetic neurons. The two main types of TLF mechanoreceptors are Ruffini and Pacini corpuscles. ${ }^{21}$ The stimulation of Ruffini receptors lowers sympathetic nervous system activity. ${ }^{22}$ Sensory fibers in the TLF have interstitial receptors. Interstitial receptors can act as both mechanoreceptors and pain receptors. Besides, the interstitial receptors regulate blood flow via a close connection with the autonomic nervous system. ${ }^{23}$ The stimulation of these receptors could lead to pain and autonomic changes. $^{24}$ Accordingly, the blockage of these receptors by local anesthetics could provide somatic and visceral analgesic effects. ${ }^{20,23}$

There is no consensus on the volume and concentration of local anesthetics used in the QLB. The suggested effective volume of local anesthetics for the QLB is at least $20 \mathrm{~mL}$ at one site. ${ }^{25}$ We used $0.25 \%$ ropivacaine $25 \mathrm{~mL}$ [62.5 mg] in this study according to the literature. ${ }^{19}$ This dose is much less than the recommended highest dose of $225 \mathrm{mg}^{26}$

Preventive analgesia using preincisional analgesic intervention could reduce postoperative pain and prevent central sensitization by blocking intraoperative noxious stimuli. $^{27}$ Therefore, we believe that preincisional QLB could prevent central sensitization and have a beneficial effect on the outcome.

There are some limitations to our study. First, we did not examine the sensory block area after QLB. We used ultrasonography to administer the QLB accurately, but we did not confirm QLB worked properly. We thought that examination of sensory block area could break the blindness of this study. But our results showed that our QLB effectively decreased pain and postoperative opioid consumption. Second, this study compared to placebo, not to active control. So, we need to evaluate the analgesic effect of QLB when compared to active control. 
Third, systemic local anesthetics could decrease pain. To exclude the systemic effect of ropivacaine, a comparative study of intravascular injection of ropivacaine needed. But from an ethical reason, we did not include the group in the present study. Fourth, this study was not designed to assess the quality of recovery, and the sample size may be too small to detect differences in the quality of recovery. As a result, we did not observe any significant differences in the quality of recovery.

\section{Conclusion}

The results of this study demonstrated that a preoperative unilateral QLB in laparoscopic-assisted nephrectomy successfully decreased postoperative pain and perioperative opioid consumption. This outcome suggests that a QLB could be an option for multimodal analgesia in laparoscopic nephrectomy.

\section{Data Sharing Statement}

The data that support the findings of this study will be stored in a secure repository at Kyungpook National Chilgok Hospital. Deidentified participant data and study protocol are available from the corresponding author, Jinseok Yeo. Data will be available up to 36 months after the publication date.

\section{Author Contributions}

All authors made substantial contributions to conception and design, acquisition of data, or analysis and interpretation of data; took part in drafting the article or revising it critically for important intellectual content; agreed to submit to the current journal; gave final approval of the version to be published; and agree to be accountable for all aspects of the work.

\section{Funding}

We did not receive any specific funding for this work.

\section{Disclosure}

The authors report no conflicts of interest in this work.

\section{References}

1. White PF, Kehlet H. Improving postoperative pain management: what are the unresolved issues? Anesthesiology. 2010;112(1):220-225. doi:10.1097/ALN.0b013e3181c6316e

2. Dunn MD, Portis AJ, Shalhav AL, et al. Laparoscopic versus open radical nephrectomy: a 9-year experience. $J$ Urol. 2000;164 (4):1153-1159. doi:10.1016/S0022-5347(05)67131-5

3. White PF, Kehlet $\mathrm{H}$. Improving pain management: are we jumping from the frying pan into the fire? Anesth Analg. 2007;105:10-12. doi:10.1213/01.ane.0000268392.05157.a8
4. Nimmo SM, Harrington LS. What is the role of epidural analgesia in abdominal surgery? Contin Educ Anaesth Crit Care Pain. 2014;14 (5):224-229. doi:10.1093/bjaceaccp/mkt062

5. Ueshima H, Otake H, Lin J-A. Ultrasound-guided quadratus lumborum block: an updated review of anatomy and techniques. Biomed Res Int. 2017;2017:2752876. doi:10.1155/2017/2752876

6. Tamura T, Kitamura K, Yokota S, Ito S, Shibata Y, Nishiwaki K. Spread of quadratus lumborum block to the paravertebral space via intramuscular injection: a volunteer Study. Reg Anesth Pain Med. 2018;43(4):372-377. doi:10.1097/AAP.0000000000000735

7. Ishio J, Komasawa N, Kido H, Minami T. Evaluation of ultrasound-guided posterior quadratus lumborum block for postoperative analgesia after laparoscopic gynecologic surgery. $J$ Clin Anesth. 2017;41:1-4. doi:10.1007/s00101-020-00766-x

8. Roberts GW, Bekker TB, Carlsen HH, Moffatt CH, Slattery PJ, McClure AF. Postoperative Nausea and Vomiting Are Strongly Influenced by Postoperative Opioid Use in a Dose-Related Manner. Anesth Analg. 2005;101(5):1343-1348. doi:10.1213/01.ANE.00001 80204.64588.EC

9. Baik JS, Oh A-Y, Cho CW, Shin H-J, Han SH, Ryu JH. Thoracic paravertebral block for nephrectomy: a randomized, controlled, observer-blinded study. Pain Med. 2014;15(5):850-856. doi:10.11 $11 /$ pme. 12320

10. Chin KJ, McDonnell JG, Carvalho B, Sharkey A, Pawa A, Gadsden J. Essentials of our current understanding: abdominal wall blocks. Reg Anesth Pain Med. 2017;42(2):133-183. doi:10.1097/ AAP.0000000000000545

11. Blanco R, Ansari T, Riad W, Shetty N. Quadratus lumborum block versus transversus abdominis plane block for postoperative pain after cesarean delivery: a randomized controlled trial. Reg Anesth Pain Med. 2016;41(6):757-762. doi:10.1097/AAP.000000000000 0495

12. Ökmen K, Metin Ökmen B, Topal S. Ultrasound-guided posterior quadratus lumborum block for postoperative pain after laparoscopic cholecystectomy: a randomized controlled double blind study. J Clin Anesth. 2018;49:112-117. doi:10.1016/j.jclinane.2018.06.027

13. Azawi NH, Mosholt KS, Fode M. Unilateral ultrasound-guided transversus abdominis plane block after nephrectomy; postoperative pain and use of opioids. Nephrourol Mon. 2016;8:e35356. doi:10.5812/ numonthly. 35356

14. Parikh BK, Waghmare VT, Shah VR, et al. The analgesic efficacy of ultrasound-guided transversus abdominis plane block for retroperitoneoscopic donor nephrectomy: a randomized controlled study. Saudi J Anaesth. 2013;7(1):43-47. doi:10.4103/1658-354X. 109808

15. Zhu M, Qi Y, He H, Lou J, Pei Q, Mei Y. Analgesic effect of the ultrasound-guided subcostal approach to transmuscular quadratus lumborum block in patients undergoing laparoscopic nephrectomy: a randomized controlled trial. BMC Anesthesiol. 2019;19(1):154. doi:10.1186/s12871-019-0825-4

16. Ahmed A, Fawzy M, Nasr MAR, et al. Ultrasound-guided quadratus lumborum block for postoperative pain control in patients undergoing unilateral inguinal hernia repair, a comparative study between two approaches. BMC Anesthesiol. 2019;19(1):184. doi:10.1186/s12871019-0862-z

17. Bagbanci O, Kursad H, Yayik AM, et al. Comparison of types 2 and 3 quadratus lumborum muscle blocks: open inguinal hernia surgery in patients with spinal anesthesia. Anaesthesist. 2020;69(6):397-403. doi:10.1007/s00101-020-00766-x

18. Kumar A, Sadeghi N, Wahal C, Gadsden J, Grant SA. Quadratus lumborum spares paravertebral space in fresh cadaver injection. Anesth Analg. 2017;125(2):708-709. doi:10.1213/ANE.00000000000 02245

19. Akerman M, Pejčić N, Veličković I. A Review of the Quadratus Lumborum Block and ERAS. Front Med (Lausanne). 2018;5:44. doi:10.3389/fmed.2018.00044 
20. Benetazzo L, Bizzego A, De Caro R, Frigo G, Guidolin D, Stecco C. 3D reconstruction of the crural and thoracolumbar fasciae. Surg Radiol Anat. 2011;33(10):855-862. doi:10.1007/ s00276-010-0757-7

21. Yahia L, Rhalmi S, Newman N, Isler M. Sensory innervation of human thoracolumbar fascia. An immunohistochemical study. Acta Orthop Scand. 1992;63(2):195-197. doi:10.3109/17453679209154 822

22. Tesarz J, Hoheisel U, Wiedenhöfer B, Mense S. Sensory innervation of the thoracolumbar fascia in rats and humans. Neuroscience. 2011;194:302-308. doi:10.1016/j.neuroscience.2011.07.066

23. Coote JH, Perez-Gonzalez JF. The response of some sympathetic neurones to volleys in various afferent nerves. J Physiol. 1970;208 (2):261-278. doi:10.1113/jphysiol.1970.sp009118
24. Wilke J, Schleip R, Klingler W, Stecco C. The lumbodorsal fascia as a potential source of low back pain: A narrative review. Biomed Res Int. 2017;2017(3):754-760. doi:10.1152/ajplegacy.1975.229.3.754

25. Carline L, McLeod GA, Lamb C. A cadaver study comparing spread of dye and nerve involvement after three different quadratus lumborum blocks. Br J Anaesth. 2016;117(3):387-394. doi:10.1093/bja/ aew 224

26. Rosenberg PH, Veering BT, Urmey WF. Maximum recommended doses of local anesthetics: a multifactorial concept. Reg Anesth Pain Med. 2004;29(6):564-575. doi:10.1016/j.rapm.2004.08.003

27. Vadivelu N, Mitra S, Schermer E, Kodumudi V, Kaye AD, Urman RD. Preventive analgesia for postoperative pain control: a broader concept. Local Reg Anesth. 2014;7:17-22. doi:10.2147/ LRA.S62160

\section{Publish your work in this journal}

The Journal of Pain Research is an international, peer reviewed, open access, online journal that welcomes laboratory and clinical findings in the fields of pain research and the prevention and management of pain. Original research, reviews, symposium reports, hypothesis formation and commentaries are all considered for publication. The manuscript management system is completely online and includes a very quick and fair peer-review system, which is all easy to use. Visit http:// www.dovepress.com/testimonials.php to read real quotes from published authors. 